\title{
CONTACT AND EQUIVALENCE OF SUBMANIFOLDS OF HOMOGENEOUS SPACES
}

\author{
ALEXANDRE A. M. RODRIGUES \\ Instituto de Matemática e Estatística \\ Universidade de São Paulo \\ São Paulo, Brazil \\ E-mail:aamrod@terra.com.br
}

1. Introduction. The problem of equivalence of submanifolds of homogeneous spaces of Lie groups was extensively treated by F. Cartan by his method of moving frames [2]. A basic idea of Cartan's method is that for sufficiently high $k, G$-contact of order $k$ (see $\S 4)$ implies $G$-equivalence. In other words, for each homogeneous space $M$ there exists an integer $k$, depending on the dimension $p$, such that if two submanifolds $S$ and $\bar{S}$ of same dimension $p$ have $G$-contact of order $k$, then there exists $g \in G$ such that $g S=\bar{S}$. Cartan treated several important geometrical examples and proved in each case the existence of $k$.

Essentially, Cartan's method of proving the existence of the element $g \in G$ consists in using the uniqueness of solution of a system of first order differential equations as in Frobenius theorem. Cartan's theory has been the subject of interest of a great number of authors (see for example [4], [5]). However, they all reduce the proof of the existence of the element $g \in G$ to the uniqueness of solution of a first order differential system whereas it seems more natural and geometrical to deal directly with a higher order differential system.

The notion of contact element as defined by Ehresmann [3] allows a geometrical formulation of the theorem of existence and uniqueness of solution of higher order completely integrable differential systems which is a straight forward generalization of Frobenius theorem (theorem 1). It is the uniqueness of this theorem that we use to solve the problem of $G$-equivalence. As a result, the regularity conditions on the submanifolds $S$ and $\bar{S}$, which are necessary for the theorem of equivalence to hold (theorem 3), can be given a simple and geometrical definition, valid in any homogeneous space $M$. Also, in the method of moving frames, the invariants of a submanifold $S$ of $M$ are defined attaching special higher order frames to the points of $S,[2],[5]$. These frames are constructed by subtle geometrical arguments valid for a fixed homogeneous space whereas we construct

2000 Mathematics Subject Classification: Primary 53A55; Secondary 53C30. 
the invariants of $S$ as the elements of a complete set of invariants of the orbits of $G$ acting on a manifold of higher order contact elements.

The equivalence problem may be posed for two immersions $f, h: S \rightarrow M$ of a differentiable manifold $S$. $f$ and $g$ are equivalent if there exists $g \in G$ such that $h=L_{g} \circ f$ where $L_{g}(x)=g x, x \in S$. This fixed parametrization theorem has been treated by J. A. Verderesi [7] by means of a higher order differential system defined in a manifold of jets.

The paper ends with a necessary and sufficient condition for a submanifold $S$ of $M$ to be an open set of an orbit of a Lie subgroup $K$ of $G$.

This paper is a summary of a lecture delivered at the 7th Conference on Geometry and Topology of Manifolds at Będlewo. Proofs will appear elsewhere.

2. Contact elements. All manifolds and maps considered in this paper are assumed to be differentiable of class $C^{\infty}$. If $M$ and $N$ are manifolds and $f: M \rightarrow N$ is a map, the induced map on tangent spaces at points $a \in M$ and $b=f(a) \in N$ will be denoted by $f_{a}: T_{a} M \rightarrow T_{b} N$. Given integers $p, k \geq 0, p \leq \operatorname{dim} M, J^{k, p} M$ denotes the manifold of all $k$-jets of rank $p$ whose source is the origin of $\mathbb{R}^{p}$ and whose target is any point of $M$. Let $G L^{k} \mathbb{R}^{p}$ be the Lie group of invertible $k$-jets whose source and target are at the origin of $\mathbb{R}^{p}$. By definition, a contact element of order $k$ and dimension $p$ of $M$ is an equivalence class of $J^{k, p} M$ under the equivalence relation: for $X, Y \in J^{k, p} M, X \sim Y$ if there exists $Z \in G L^{k}\left(R^{p}\right)$ such that $Y=X \circ Z$. The set of contact elements of order $k$ and dimension $p$ of $M$ is a differentiable manifold denoted by $C^{k, p}(M) . C^{0, p}(M)$ identifies naturally with $M[3]$.

For $0 \leq k^{\prime} \leq k$ there is a natural projection $\pi_{k^{\prime}}^{k}: C^{k, p}(M) \rightarrow C^{k^{\prime}, p}(M)$. If $k^{\prime}=0$, we write $\pi^{k}: C^{k, p} M \rightarrow M$ instead of $\pi_{0}^{k}$. The fiber of $C^{k, p} M$ over $a \in M$ is denoted by $C_{a}^{k, p} M$. If $p$ is the dimension of $M, C_{a}^{k, p} M$ has only one element which is denoted by $C_{a}^{k} M$ and is called the contact element of order $k$ of $M$ at the point $a \in M$.

Given a submanifold $S$ of $M, S \subset M$, and an integer $p, 0 \leq p \leq \operatorname{dim} S$, there is a natural injection of $C^{k, p} S$ into $C^{k, p} M$. If $p$ is the dimension of $S$, composing the map $a \in S \rightarrow C_{a}^{k, p} S \in C^{k, p} M$ with the injection $C^{k, p} S \rightarrow C^{k, p} M$, we define an injection $C^{k}: a \in S \rightarrow C_{a}^{k} S \in C^{k, p} M$. The image of this injection is denoted by $C^{k} S \subset C^{k, p} M$. Two submanifolds $S$ and $\bar{S}$ of $M$ of the same dimension $p$ have contact of order $k$ at a common point $a$ if $C_{a}^{k} S=C_{a}^{k} \bar{S}$.

3. Completely integrable differential systems of higher order. A differential system of order $k \geq 1$ and dimension $p$ defined over a manifold $M$ is a submanifold $\Omega^{k}$ of $C^{k, p} M$ such that the projection $\pi^{k}: \Omega^{k} \rightarrow M$ is of rank equal to the dimension of $M$. An integral manifold of $\Omega^{k}$ is a submanifold $S$ of $M$ of dimension $p$ such that $C_{x}^{k} S \in \Omega^{k}$ for all $x \in S$. For $X \in C^{k, p} M$, let $F_{X}$ be the fiber of $X$ by the projection $\pi_{k-1}^{k}: C^{k, p} M \rightarrow C^{k-1, p} M$. The symbol $\sigma(X)$ of $\Omega^{k}$ at the point $X \in \Omega^{k}$ is by, definition, the vector space

$$
\sigma(X)=T_{X} \Omega^{k} \cap T_{X} F_{X}
$$

Let $X^{k+1} \in C^{k+1, p} M, X^{k}=\pi_{k}^{k+1}(X)$, and let $S$ be a submanifold of $M$ such that $X^{k+1}=C_{a}^{k+1} S, a \in S$. Then, $C_{X^{k}}^{1}\left(C^{k} S\right)$ depends only on $X^{k+1}$ and not on the choice 
of $S$. Hence, there is a natural imbedding

$$
\bar{\Lambda}^{k, 1}: C^{k+1, p} M \rightarrow C^{1, p}\left(C^{k, p} M\right)
$$

which maps $X^{k+1}$ into $C_{X^{k}}^{1}\left(C^{k} S\right)$. By definition, the first prolongation of the differential system $\Omega^{k}$ is the subset $\Omega^{k, 1}$ of $C^{k+1, p} M$ defined by

$$
\Omega^{k, 1}=\left(\bar{\Lambda}^{k, 1}\right)^{-1}\left[C^{1, p}\left(\Omega^{k}\right) \cap \bar{\Lambda}^{k, 1}\left(C^{k+1, p} M\right)\right] .
$$

Since $\pi_{k}^{k+1}=\pi_{0}^{1} \circ \Lambda^{k, 1}$, it follows that $\pi_{k}^{k+1}$ maps $\Omega^{k, 1}$ into $\Omega^{k}$. If $S$ is an integral manifold of $\Omega^{k}$ then, $C_{x}^{k+1} S \in \Omega^{k, 1}$ for every $x \in S$. Hence, a necessary condition for the existence of an integral manifold of $\Omega^{k}$ going through every point of $\Omega^{k}$ is that the projection $\pi_{k}^{k+1}: \Omega^{k, 1} \rightarrow \Omega^{k}$ be surjective.

THEOREM 1. Let $\Omega^{k} \subset C^{k, p} M$ be a differential system of order $k \geq 1$ and let $X \in \Omega^{k}$ be a contact element such that

1) $\sigma(X)=\{0\}$;

2) The image of $\Omega^{k, 1}$ by the projection $\pi_{k}^{k+1}: \Omega^{k, 1} \rightarrow \Omega^{k}$ is a neighborhood of $X$ in $\Omega^{k}$.

Then, there exists an integral manifold $S$ of $\Omega^{k}$ such that $X \in C^{k} S$. Moreover, if $S$ and $S^{\prime}$ are integral manifolds of $\Omega^{k}$ such that $X \in C^{k} S \cap C^{k} S^{\prime}$, there exists a set $W$ which is an open neighborhood of $X$ in $C^{k} S$ and $C^{k} S^{\prime}$.

Theorem 1 is a geometrical version of the theorem of existence and uniqueness of solutions of completely integrable systems of partial differential equations of order $k \geq 1$. Taking suitable coordinates in $C^{k+1, p} M$ and $C^{k, p} M$, the existence of integral manifolds of $\Omega^{k}$ reduces to the existence of solutions of a completely integrable system of partial differential equations [6].

4. Contact of submanifolds. Let $G$ be a Lie group acting transitively on the manifold $M$. Two submanifolds $S$ and $\bar{S}$ of $M$ of same dimension $p$, have $G$-contact of order $p$ at points $a \in S$ and $\bar{a} \in \bar{S}$ if there exists $g \in G$ such that $g a=\bar{a}$ and $g S$ and $\bar{S}$ have contact of order $k$ at the point $\bar{a} . S$ and $\bar{S}$ have $G$-contact of order $k \geq 0$ if there exists a diffeomorphism $\phi: S \rightarrow \bar{S}$ such that for all $x \in S, S$ and $\bar{S}$ have contact of order $k$ at points $x$ and $\phi(x)=g(x) x$. We say in this case that $\phi$ makes contact of order $k$ of $S$ onto $\bar{S} . S$ and $\bar{S}$ are $G$-equivalent if there exists $g \in G$ such that $g S=\bar{S} . S$ and $\bar{S}$ are locally $G$-equivalent at points $a \in S$ and $\bar{a} \in \bar{S}$ if there are open neighborhoods of $a$ and $\bar{a}$ in $S$ and $\bar{S}$ which are $G$-equivalent.

The action of $G$ on $M$ extends to an equivariant action on the manifold $C^{k, p} M$ of contact elements of order $k$ and dimension $p$ of $M$. For a point $x \in M$, let $C_{x}^{k} S, G_{x}^{k}$ and $d^{k}(x)$ denote respectively the contact element of order $k$ of $S$ at the point $x$, the isotropy subgroup of $G$ at the point $C_{x}^{k} S$ and the dimension of $G_{x}^{k}$. We call $G_{x}^{k}$ the isotropy subgroup of order $k$ of the point $x$ of $S$. Put $X=C_{x}^{k} S$ and let $h^{k}(x)$ be the dimension of the vector space $T_{X}(G X) \cap T_{X} C^{k} S$ where $C^{k} S$ is the submanifold of $C^{k, p} M$ of all contact elements of order $k$ of $S$ and $T_{X}(G X)$ and $T_{X} C^{k} S$ are the tangent spaces of the orbit $G X$ and of $C^{k} S$ at the point $X$.

For $k^{\prime} \leq k, d^{k}(x) \leq d^{k^{\prime}}(x)$ and $h^{k}(x) \leq h^{k^{\prime}}(x)$. Hence, there exists an integer $k \geq 1$ such that $d^{k}(x) d^{k-1}(x)$ and $h^{k}(x)=h^{k-1}(x)$. We say that $a \in S$ is a $k$-regular point of 
$S$ under the action of $G$ if there exists $k \geq 1$ such that

1) $d^{k}(a)=d^{k-1}(a)$ and $h^{k}(a)=h^{k-1}(a)$;

2) $d^{k}(x)$ and $h^{k}(x)$ are constant for $x$ varying in a neighborhood of $a$ in $S$.

The order of $a$ is the least integer satisfying conditions above. If $a$ is a $k$-regular point of $S$ then $g a$ is a $k$-regular point of $g S$.

THEOREM 2. Let $S, \bar{S}$ be two submanifolds of $M$ of same dimension $p$. Let $a \in S$ and $\bar{a} \in \bar{S}$ be two points. Assume that $\bar{a}$ is a k-regular point of $\bar{S}$ and that there exists a continuous map $\varphi: V \rightarrow G$, defined in a neighborhood $V$ of a in $S$, such that $\varphi(a) . a=\bar{a}$, $\varphi(x) . x \in \bar{S}$ and $\varphi(x) . C_{x}^{k} S=C_{\varphi(x)}^{k} \bar{S}$ for all $x \in V$. Then, there exist open neighborhoods $W$ and $\bar{W}$ of $a$ and $\bar{a}$ in $S$ and $\bar{S}$ which are G-equivalent.

The proof of theorem 2 is based on the uniqueness statement of theorem 1 .

We assume in theorems $3,4,5,6,8$ that the action of $G$ on $\mathrm{M}$ is proper and that $H$ is a closed subgroup of $G$. Let $L$ be the union of all $G$-orbits of $C^{k, p} M$ of type $H$ that is, orbits whose isotropy subgroups are conjugate to $H$. Denote by $L / G$ the quotient space of $L$ by the orbits and by $\pi: L \rightarrow L / G$ the natural projection. It is known [1] that $L$ and $L / G$ are differentiable manifolds and that $(L, L / G, \pi)$ is a locally trivial fiber bundle.

Let $f: S \rightarrow \bar{S}$ be a diffeomorphism such that $S$ and $\bar{S}$ have $G$-contact of order $k \geq 1$ at corresponding points $x \in S$ and $\bar{x}=f(x) \in \bar{S}$ and let $a \in S$ and $\bar{a}=f(a) \in \bar{S}$ be two points. Considering suitable cross sections of the fiber bundle $(L, L / G, \pi)$, one can prove the existence of a neighborhood $V$ of $a$ in $S$ and of a differentiable map $\varphi: V \rightarrow G$ such that $\varphi(x) \cdot x=f(x)$ and $\varphi(x) \cdot C_{x}^{k} S=C \bar{x} \bar{S}$. Hence, theorem 2 can be restated as follows:

THEOREM 3. Assume that the action of $G$ on $M$ is proper and that there exists $k \geq 1$ such that

1. $\bar{a} \in \bar{S}$ is a k-regular point.

2. The isotropy subgroups of $C_{\bar{x}} \bar{S}$ are conjugate in $G$ for all $\bar{x} \in \bar{S}$.

3. There exists a diffeomorphism $f: S \rightarrow \bar{S}$ such that $S$ and $\bar{S}$ have $G$-contact of order $k$ at corresponding points.

Let $a \in S$ be such that $f(a)=\bar{a}$. Then $S$ and $\bar{S}$ are locally $G$-equivalent at points $a$ and $\bar{a}$.

THEOREM 4. Assume that $S$ and $\bar{S}$ are connected and that there exists an integer $k \geq 1$ such that:

1. $\bar{x} \in \bar{S}$ is a k-regular point of $\bar{S}$ and $h^{k}(\bar{x})=0$ for all $\bar{x} \in \bar{S}$.

2. The isotropy subgroups of $C_{\bar{x}} \bar{S}$ are conjugate in $G$ for all $\bar{x} \in \bar{S}$.

3. There exists a diffeomorphism $f: S \rightarrow \bar{S}$ such that $S$ and $\bar{S}$ have $G$-contact of order $k$ at corresponding points.

Then, $f$ is the restriction to $S$ of the translation by an element $g$ of $G: f=L_{g} \mid S$.

Consider again the fiber bundle $(L, L / G, \pi)$. There exists a finite number of real valued differentiable functions $\tilde{\rho}_{i}, 1 \leq i \leq r$, defined in $L$, such that two contact elements $X, \bar{X} \in L$ are in the same fiber of $L$ if and only if $\tilde{\rho}_{i}(X)=\tilde{\rho}_{i}(\bar{X}), 1 \leq i \leq r$. Given a submanifold $S$ of $M$ of dimension $p$, and assuming that the orbits of $C_{x}^{k} S$ are of type $H$ for all $x \in S$, one can pull back the functions $\tilde{\rho}_{i}$ by the map $\sigma^{k}: x \in S \rightarrow C_{x}^{k} S \in L$. 
The set of functions $\rho_{i}=\tilde{\rho}_{i} \circ \sigma^{k}, 1 \leq i \leq r$, is a complete set of $G$-invariants of order $k$ of the submanifold $S$ of $M$. Often the invariants can be defined in a natural way and have deep geometrical meaning as for instance, the curvature and torsion of curves and the principal curvatures of surfaces in $\mathbb{R}^{3}$.

Assuming that the isotropy subgroups of $C_{x}^{k} S$ and $C_{\bar{x}}^{k} \bar{S}$ are of type $H$ for all $x \in S$ and $\bar{x} \in \bar{S}$, complete sets of invariants of order $k, \rho_{i}$ and $\bar{\rho}_{i}$ can be defined in $S$ and $\bar{S}$. The condition $h^{k}(\bar{x})=0$ in theorem 4 is then clearly equivalent to stating that the rank of differentials $d \bar{\rho}_{i}, 1 \leq i \leq r$, is $p$ at every point $\bar{x} \in \bar{S}$ One can then restate theorems 3 and 4 in the following way.

THEOREM 5. Let $\bar{a} \in \bar{S}$ be a k-regular point of $\bar{S}, k \geq 1$. Assume the following conditions are satisfied:

1. The isotropy subgroups of $C_{x}^{k} S$ and $C_{\bar{x}}^{k} \bar{S}$ are conjugate for all $x \in S$ and $\bar{x} \in S$.

2. There exists a diffeomorphism $f: S \rightarrow \bar{S}$ such that

$$
\bar{\rho}_{i}=\rho_{i} \circ f, 1 \leq i \leq r .
$$

Then, $S$ and $\bar{S}$ are locally G-equivalent at points $a=f^{-1}(\bar{a})$ and $\bar{a}$.

TheOREM 6. Let $S, \bar{S}$ be two connected submanifolds of $M$ and let $k \geq 1$ be such that

1. Every point $\bar{x} \in \bar{S}$ is k-regular.

2. The isotropy subgroups of $C_{x}^{k} S$ and $C_{\bar{s}}^{k} \bar{S}$ are conjugate for all $x \in S$ and $\bar{x} \in S$.

3. There exists a diffeomorphism $f: S \rightarrow \bar{S}$ such that

$$
\rho_{i}=\bar{\rho}_{i} \circ f, 1 \leq i \leq r .
$$

4. The rank of differentials $d \bar{\rho}_{i}, 1 \leq i \leq r$, is $p$ at every point $\bar{x} \in \bar{S}$.

Then, $f$ is the restriction to $S$ of the left translation by an element of $G: f=\mathcal{L}_{g} \mid S$.

Let us assume that $S$ is an open set of an orbit of a Lie subgroup $K$ of $G$. Then, $h^{k}(x)=p$ and the isotropy subgroups of $C_{x}^{k}$ are conjugate for all $x \in S$ and $k \geq 0$. Hence there exits $k \geq 1$ such that every $x \in S$ is a k-regular point of $S$. Conversely,

THEOREM 7. A necessary and sufficient condition for a connected submanifold $S$ of $M$ to be an open set of an orbit of a Lie subgroup $K$ of $G$ is the existence of $k \geq 1$ such that for all $x \in S, x$ is a k-regular point of $S$ and $h^{k}(x)=p$.

Assuming that the action of $G$ on $M$ is proper and that the isotropy subgroups of order $k$ of points of $S$ are conjugate, a complete set of invariants of order $k$ can be defined on $S$. Clearly, $h^{k}(x)=p$ for every $x \in S$ if and only if the invariants are constant on $S$. Therefore, the following corollary to theorem 7 holds.

THEOREM 8. Assume that the action of $G$ on $M$ is proper and that $S$ is connected. Assume also that for some integer $k \geq 1$, every point of $S$ is k-regular and all isotropy subgroups of order $k$ of points of $S$ are conjugate. Then, a necessary and sufficient condition for $S$ to be an open set of an orbit of a Lie subgroup of $G$, is that the invariants of order $k$ of $S$ be constant. 


\section{References}

[1] N. Bourbaki, Groupes et algèbres de Lie, Chapitre 9, Masson, Paris, 1982.

[2] E. Cartan, Théorie des groupes finis et continues et la géométrie différentielle traitées par la méthode du repère mobile, Gauthier-Villars, Paris, 1937.

[3] C. Ehresmann, Introduction à la théorie des structures infinitésimales et des pseudo-groupes de Lie, in: Coll. Géométrie Différentielle, Strasbourg, 97-117.

[4] P. Griffiths, On Cartan's methods of Lie groups and moving frames as applied to uniqueness and existence questions in differential geometry, Duke Math. J. 41 (1974), 775-814.

[5] G. R. Jensen, Higher Order Contact of Submanifolds of Homogeneous Spaces, Lecture Notes in Math. 610, Springer, Berlin, 1977.

[6] M. Kuranishi, Lectures on Involutive Systems of Partial Differential Equations, Publicações da Sociedade de Matematica de São Paulo, 1967.

[7] J. A. Verderesi, Contact et congruence de sous variétés, Duke Math. J. 49 (1982), 513-515. 